\title{
Diagnóstico de riesgos ergonómicos que afectan el desarrollo de las actividades de docencia y administración
}

\section{Riesgos ergonómicos en las actividades de docencia y administración}

Ing. Sandy Raúl Chun Molina Mg. Sc. ${ }^{(1)}$

Ing. Enrique Javier Macías Arias.

Ing. Gregorio Isoldo Mendoza Cedeño Mg. Eds. ${ }^{(3)}$

Ing. Carlos Javier Rodríguez Vera. ${ }^{(4)}$

${ }^{(1)}$ Instituto Tecnológico Superior Paulo Emilio Macías, Ecuador

${ }^{(2)}$ Instituto Tecnológico Superior Portoviejo, Ecuador

${ }^{(3)}$ Instituto Tecnológico Superior Paulo Emilio Macías, Ecuador

${ }^{(4)}$ Instituto Tecnológico Superior Paulo Emilio Macías, Ecuador

Contacto: $\underline{\text { itspem.rchum@gmail.com }}$

Receptado: 10/09/2016 Aceptado: 25/11/2016

\section{Resumen}

El presente trabajo tiene como propósito conocer la situación actual del trabajo en la oficina respecto a las condiciones ergonómicas y los muchos factores tanto de diseño de mobiliario (mesa, silla, pantallas de visualización de datos) como de factores ambientales (iluminación, ruido, entre otros), que afectan al personal docente y administrativo en las instituciones públicas y privadas de educación superior, que realizan grandes esfuerzos por mejorar la implementación de mobiliarios, incorporando equipos de cómputo modernos o actualizándolos, sin embargo hay una parte importante que han pasado por alto: La importancia de trabajar en un ambiente ergonómico adecuado, donde no se ha contemplado en su diseño criterios ergonómicos, lo cual puede generar a corto y mediano plazo malestares físicos y emocional es en el personal que lo ocupa, disminuyendo la eficacia y productividad laboral. Dada la situación expuesta, se generó el interés por realizar el presente trabajo en una institución educativa de nivel superior, con la finalidad de conocer la situación laboral que prevalece en ésta. En conclusión un adecuado diseño de las instalaciones (oficina, climatización, iluminación, y acondicionamiento acústico), una correcta selección del equipamiento que se compra (sillas, mesas de trabajo, equipos informáticos, entre otros) y una correcta organización en las tareas permitirá prevenir una buena parte de las molestias de tipo postural, condiciones ambientales correctas y problemas de naturaleza psicosocial. Finalmente, todas las acciones antes mencionadas 
pueden resultar ineficaces si se deja de lado la necesaria labor de formación e información de los trabajadores.

Palabras claves: Enfermedades, psicosocial, mobiliario, ergonómico, acondicionamiento acústico.

\title{
Diagnosis of ergonomic risks that affect the development of teaching and administrative activities
}

Riesgos ergonómicos en las actividades de docencia y administrativa

\begin{abstract}
The purpose of this work is to know the current labor situation in the office regarding of ergonomic conditions and of the many factors of furniture design (table, chair, data visualization screens) and environmental factors (lighting, noise, others), which affect the teaching and administrative staff in public and private institutions of higher education, who make great efforts for improve the implementation of furniture, incorporating modern computer equipment or updating them, however there is an important part that have been ignored : The importance this work is an appropriate ergonomic environment, where ergonomic criteria have not been considered in its design, which can generate short and medium term physical and emotional discomfort in the personnel occupying it, reducing efficiency and labor productivity. Given the situation exposed, the interest was generated to carry out the present work in an educational institution of higher level, with the purpose of knowing the labor situation that prevails in this one. In conclusion, an adequate design of the facilities (office, air conditioning, lighting, and acoustic conditioning), a correct selection of the equipment that is bought (chairs, work tables, computer equipment, among others) A good part of the postural type discomfort, correct environmental conditions and problems of a psychosocial nature. Finally, all the above-mentioned actions can be ineffective if the necessary training and information of the workers is left aside.
\end{abstract}

Keywords: Diseases, psychosocial, furniture, ergonomic, acoustic conditioning.

\section{Introducción}

El uso de la tecnología en las instituciones educativas se ha convertido en una herramienta fundamental de apoyo a las actividades de docencia, administrativa y de investigación.

Los avances y progresos por entrar a una vanguardia educativa, no sólo es proporcionara los usuarios herramientas con alta tecnología, se debe contemplar contar con áreas de trabajo 
diseñadas bajo criterios ergonómicos, que le permita al usuario desempeñar sus actividades, sin que se vea afectada su salud y por lo tanto se vea afectado su desempeño laboral. (Donald, 2003)

En la mayor parte de los casos, la postura que adoptan los trabajadores conduce a la aparición de las lesiones ergonómicas : cefaleas, dolores de espalda, molestias cervicales, lumbalgias, agarrotamientos musculares... los esfuerzos de mano, muñeca, codo y hombro traen consigo tendinitis, tenosinovitis, entre otros, a consecuencia de movimientos rápidos, forzados y repetidos que inflaman las articulaciones. Denominado "Síndrome de Tensión Repetitiva" y herencia de los adelantos tecnológicos, conforma un cuadro clínico estrechamente ligado al manejo de video terminales. (Vélez, 2002)

\section{Evolución de la ergonomía}

"La ergonomía como ciencia o disciplina integrada surgió hace algunos decenios: Sin embargo, empíricamente data de los tiempos de la sociedad primitiva. El término ergonomía proviene de las palabras griegas ergon (trabajo) y nomos (la ley, norma o doctrina (1))" (Ramirez, 2010); la primera referencia a la ergonomía aparece citada en el libro del polaco Wojciech Jastrzebowki (1857) titulado "Compendio de Ergonomía” o de la ciencia del trabajo basado en verdades tomadas de la naturaleza, que según la traducción de Pacaud (1974) dice: "para empezar un estudio científico del trabajo y elaborar una concepción de la ciencia del trabajo, no debemos supeditarla en absoluto a otras disciplinas científicas, para que sea ciencia del trabajo, simultáneamente a nuestras facultades físicas, estéticas, racionales y morales". (Bonilla, 2001)

\section{Enseñanza por medio de las computadoras}

Los primeros esfuerzos por automatizar en parte el proceso enseñanza aprendizaje se pueden encontrar en el uso de las máquinas de enseñanza de Sídney Pressey, profesor de un curso introductorio masivo de psicología educativa en la Universidad de Ohio. Motivado por el posible ahorro de tiempo diseñó una máquina que se parecía al carro de una máquina de escribir, con cuatro teclas y una ventana larga por la cual se podría ver un marco con una pregunta y cuatro posibles respuestas. (Hérnandez, 2016)

\section{Antropometría y dimensiones del puesto}


La arquitectura y el urbanismo son los escenarios donde nos desarrollamos y sólo tienen sentido en función a sus usuarios: las personas. En el diseño de espacios, equipamiento y mobiliario, se debe tener en cuenta la diversidad de características físicas, destrezas y habilidades de los usuarios, conciliando todos los requerimientos especiales que esto implica. (Rodríguez, 2006.)

Cuando se diseña y construye pensando en las personas con discapacidad, se logran entornos accesibles para todos. Las dimensiones de los espacios habitables, necesarios para el desplazamiento y maniobra de personas que utilizan sillas de ruedas, muletas, andaderas, bastones y perros guía, tienen su fundamento en la antropometría y características propias de cada ayuda técnica.

La accesibilidad se logra pensando en los espacios y en los recorridos, como parte de un sistema integral. De nada sirve un baño adecuado, si llegar a él implica salvar escalones o atravesar puertas angostas. Las disposiciones administrativas son un complemento necesario a los inmuebles accesibles. No es insólito encontrar establecimientos adecuados, en los que está prohibida la entrada con animales en general, sin hacer la distinción entre mascotas y perros guía.(Rodríguez, Arqhys, 2006)

Dado que las posturas y los movimientos naturales son indispensables para un trabajo eficaz, es importante que el puesto de trabajo se adapte a las dimensiones corporales del operario, no obstante, ante la gran variedad de tallas de los individuos éste es un problema difícil de solucionar.

Para el diseño de los puestos de trabajo, no es suficiente pensar en realizarlos para personas de talla media (50 percentil), es más lógico y correcto tener en cuenta a los individuos de mayor estatura para acotar las dimensiones, por ejemplo del espacio a reservar para las piernas debajo de la mesa, y a los individuos de menor estatura para acotar las dimensiones de las zonas de alcance en plano horizontal. (Percentiles 95 - 5). (R.C., 2006)

\section{Iluminación}

La iluminación es la cantidad de luminosidad que se presenta en el sitio de trabajo del empleado. No se trata de iluminación general sino de la cantidad de luz en el punto focal del trabajo. De este modo, los estándares de iluminación se establecen de acuerdo con el tipo de tarea visual que el empleado debe ejecutar: cuanto mayor sea la concentración visual del empleado en detalles y minucias, más necesaria será la luminosidad en el punto focal del trabajo. La iluminación deficiente ocasiona fatiga a los ojos, perjudica el sistema nervioso, 
ayuda a la deficiente calidad del trabajo y es responsable de una buena parte de los accidentes de trabajo. (Montes, 2000)

Las recomendaciones de iluminación en oficinas son de 300 a 700 luxes, para que no reflejen se puede controlar con un reóstato. El trabajo que requiere una agudeza visual alta y una sensibilidad al contraste necesita altos niveles de iluminación. El trabajo fino y delicado debe tener una iluminación de 1000 a 10000 luxes.

\section{Materiales y Métodos}

En cuanto al tipo de exploración que se utilizó en el proyecto es la de investigación de campo, que se apoya en informaciones donde se originan encuestas, mediante este tipo de investigación se utiliza el método de análisis descriptivo, se logra caracterizar un objeto de estudio o una situación concreta, señalar sus características y propiedades. La recolección de datos se efectuó pormedio de encuestas que fueron aplicadas a 65 personas entre docentes y administrativos del Instituto Tecnológico Superior Paulo Emilio Macías (ITSPEM), que se encuentran en edades de 26 a 58 años. Se recopiló dicha información necesaria para realizar el diagnóstico del trabajo investigativo.

\section{Resultados}

En este apartado me centraré en el desarrollo global sobre el conjunto de la muestra objeto de investigación, que quedó configurada por 65 personas (65encuestas), lo que supone un 85\% del total del personal existentes en el ITSPEM. Esto quiere decir que el índice de respuesta obtenido ha sido representativo.

El 97.50\% del personal encuestado laboran la jornada a tiempo completo, es decir 8 horas diarias, 40 horas semanales.

Los encuestados presentan un $100 \%$ de manejo de computadoras como herramientas de enseñanza.

Un porcentaje alto de los encuestados destacan la poca aplicación de cultura ergonómica en la institución.

Un 93\% de los encuetados menciona que no posee un sistema eficiente de iluminación directa en el momento de realizar su trabajo.

Un $80 \%$ de los encuestados mencionan tener problemas posturales y cervicales al finalizar su jornada de labores. 
El mobiliario utilizado por el personal docente y administrativo de la institución no posee un mecanismo auto regulable de acuerdo a la contextura del usuario que lo utiliza.

En lo referente a las mesas de trabajo de la sala de docentes asignada para la planificación de actividades de preparación de clases, vinculación e investigación no cumple con las características que la normativa establece.

Problemas de salud generados por riesgos ergonómicos en áreas de trabajo con pantallas de visualización de datos

El trabajo en vídeo terminales, es decir, pantallas de visualización de datos, (PVD), produce problemas de orden físico y fisiológico en los operadores. Algunos de los síntomas producidos son relativamente menores y desaparecen cuando se suprime el causante del inconveniente, pero otros en cambio, combinados con diversos factores producen problemas no tan simples, son más significativos y pueden llevar el riesgo a lesiones graves o agravar las existentes. (Mondelo P., 2002)

No todos los problemas de salud son el resultado de los efectos de las pantallas de datos, si no muchos de ellos son el resultado de una mala configuración del puesto de trabajo, independientemente si en él se trabaja con una PVD o no. No obstante la mayoría de los problemas entre los operadores, son de origen profesional, motivo por el cual se pretende en el futuro reglamentar el trabajo en las video terminales de manera tal que no haya lugar a dudas sobre los riesgos para la salud.

Los problemas más comunes son del tipo visual; para un mejor estudio los vamos a analizar por separado.

\section{Problemas Visuales}

"El problema más común de orden visual en los centros de cómputos es el cansancio visual (asthenopía), que trae como consecuencia la disminución de la agudeza visual, dolores de cabeza, cervicales, irritación de la vista entre otras causas” (Mondelo P., 2002).

Hay personas que sostienen que el trabajo en vídeo terminales producen una disminución de la visión a largo plazo, si bien no hay pruebas que demuestren científicamente la veracidad del video terminales que tienen problemas visuales previos, sufre con la labor una mayor fatiga, que las personas las personas con buena salud. 
Dado como ya se acota antes, los defectos de la vista se agrava con la edad, dicho problema se plantea cada vez con mayor frecuencia en los operadores de mayor edad. Por esta razón, es necesario que los acuerdos que se hagan con los operadores especifiquen un régimen de controles regulares da la vista, para evitar que los defectos se agraven.

\section{Síntomas más frecuentes de fatiga visual}

Irritación de los ojos.

Dolores o presión en los globos oculares.

Dolores de cabeza.

Ojos más sensibles a la luz.

\section{Porcentaje de trastornos visuales en usuarios de PCD}

Vista cansada $72 \%$

Picazón, ardor 64\%

Dolor o presión de los globos oculares $54 \%$

Desdoblamiento de imagen $24 \%$

Ojos más sensibles a la luz 64\%

\section{Problemas Músculo-Esqueléticos (Lesiones Ocasionadas por Movimientos Repetitivos)}

Existen lesiones que se producen como consecuencia de repetir el mismo movimiento constantemente, un caso típico es la tendosinovitis y el síndrome del túnel carpiano, que afectan de manera general a los dedos, las manos, las muñecas y/o los brazos, que se da en los operadores de entrada de datos y en los tipistas de todo tipo, los cuales utilizan constantemente el teclado para entrar datos a almacenar en los sistemas informáticos a gran velocidad. (TeensHealth, 2016)

\section{Porcentaje de síntomas músculo-esqueléticos en usuarios}

Dolor de espalda $76 \%$

Dolor de nuca $55 \%$ 
Dolor lumbar 30\%

Dolor en otras articulaciones muñecas) $31 \%$

Pesadez de miembros $38 \%$

Las lesiones en la espalda baja son uno de los problemas de mayor recurrencia a los que se enfrenta la medicina preventiva en el área industrial, y en un estudio conducido por el Instituto Nacional para la Seguridad y Salud Ocupacional (NIOSH) sobre la exposición ocupacional entre 1981 y 1983, encontró que aproximadamente el 30\% de la fuerza laboral de la industria norteamericana estaba involucrado de alguna forma en trabajos donde se exponen a lesiones y daños físicos asociados con el manejo manual de materiales. (Ocupacional, 2016)

\section{Región cervical y nuca}

A nivel de la región cervical y nuca, los dolores vienen condicionados por los continuos movimientos de la cabeza del operador, y por la existencia de distancias distintas (documentos, pantalla, teclado, uso indebido del teléfono). Si el diseño del puesto no cumple los mínimos requisitos ergonómicos, los movimientos de la cabeza del operador tendrán que ser de mayor amplitud.

\section{Región lumbar}

A nivel lumbar, los trastornos son generados por una mala acomodación entre el trabajador y el puesto, se ven obligados a mantener su columna vertical erguida y recta, haciendo desaparecer las curvas fisiológicas, lo que se consigue a través de una contracción isométrica de los músculos dorsales. La consecuencia es el agotamiento y el dolor.

\section{Discusión}

\section{Skinner}

F. B. Skinner, profesor de la Universidad de Harvard, quien sentó las bases psicológicas para la llamada enseñanza programada. Skinner desarrolló sus principios de análisis de la conducta y sostuvo que era indispensable una tecnología de cambio de la conducta. Atacó la costumbre contemporánea de utilizar el castigo para cambiar la conducta y sugirió que el uso de recompensas y refuerzos positivos de la conducta correcta era más atractivo desde el punto de vista social y pedagógicamente más eficaz. Además definió la enseñanza como la modificación o moldeado de las respuestas emitidas conductualmente en vez de la transmisión del conocimiento. Opinó que el salón de clase no era un ambiente apropiado para dar refuerzo 
adecuado y sugirió las máquinas de enseñanza como una vía más práctica para lograrlo. Skinner adoptó las máquinas de Pressey, con algunas modificaciones para que no estuvieran restringidas a la selección de respuestas alternativas, y aseveró que el refuerzo intermitente y frecuentemente de respuestas correctas era la causa de la alteración de la conducta. Por este motivo organizó la instrucción en pequeñas unidades llamadas marcos (frames). Después de cada marco que presentaba información al estudiante se le pedía que diera una respuesta a una pregunta que se comparaba con la respuesta correcta o deseable. Si coincidían, se daba un refuerzo. En vista de que los errores no generaban refuerzos se trataban de evitar, lo cual se lograba haciendo que los marcos fueran muy cercanos entre sí y frecuentemente se daban sugerencias para que con más facilidad el estudiante diera respuestas correctas. (ANUIES., 1997. )

\section{Altura del plano de trabajo}

La determinación de la altura del plano de trabajo es muy importante para la concepción de los puestos de trabajo, ya que si ésta es demasiado alta tendremos que levantar la espalda con el consiguiente dolor en los homóplatos, si por el contrario es demasiado baja provocaremos que la espalda se doble más de lo normal creando dolores en los músculos de la espalda. Es pues necesario que el plano de trabajo se sitúe a una altura adecuada a la talla del operario, ya sea en trabajos sentados o de pie. (R.C., 2006)

Para un trabajo sentado, la altura óptima del plano de trabajo estará en función del tipo de trabajo que vaya a realizarse, si requiere una cierta precisión, si se va a utilizar máquina de escribir, si hay exigencias de tipo visual o si se requiere un esfuerzo mantenido.

Si el trabajo requiere el uso de computadora una gran libertad de movimientos es necesario que el plano de trabajo esté situado a la altura de los codos; el nivel del plano de trabajo nos lo da la altura de la máquina, por lo tanto la altura de la mesa de trabajo deberá ser un poco más baja que la altura de los codos.

Si por el contrario el trabajo es de oficina, leer y escribir, la altura del plano de trabajo se situará a la altura de los codos, teniendo presente elegir la altura para las personas de mayor talla ya que los demás pueden adaptar la altura con sillas regulables.

Figura $\mathrm{N}^{\circ} 1$ : Altura del plano para puestos de trabajo sentado medido en mm
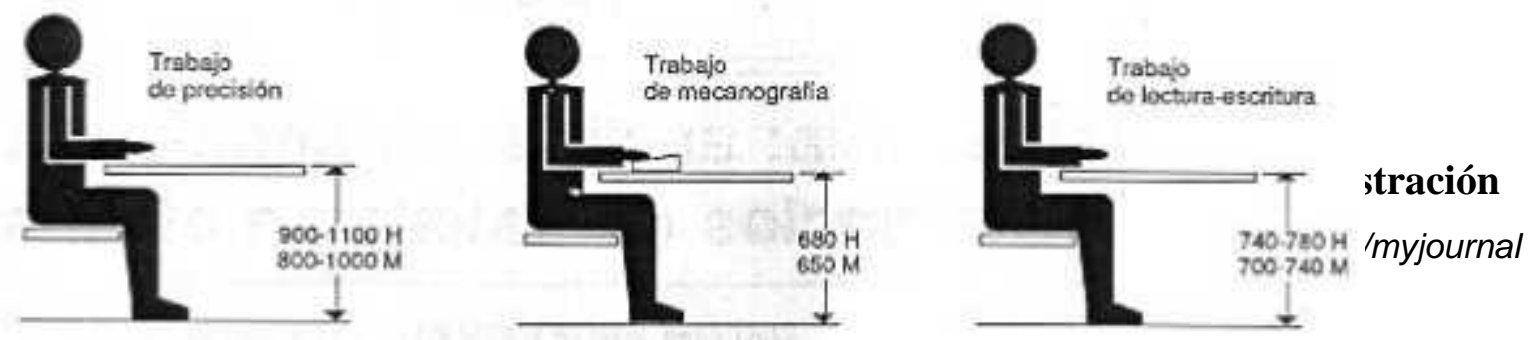
Fuente: Chavarría, 2005.

Elaborado por: Autores de investigación

\section{Espacio reservado para las piernas}

En este apartado se pretende definir si el espacio reservado para las piernas permite el confort postural del operario en situación de trabajo. Las dimensiones mínimas de los espacios libres para piernas, serán las que se dan en la figura 2.

Figura $\mathrm{N}^{\circ}$ 2: Medidas de emplazamiento para las piernas en puestos de trabajo sentado.

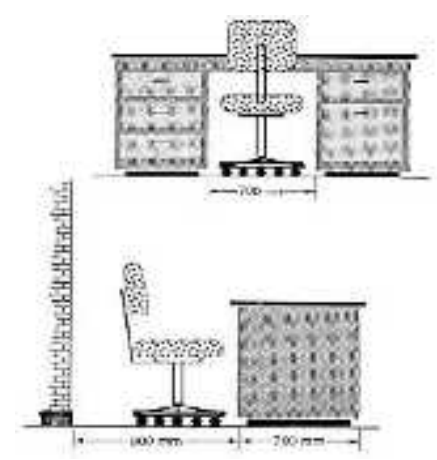

Fuente: Chavarría, 2005.

Elaborado por: Autores de investigación

\section{Zonas de alcance óptimas del área de trabajo}

Una buena disposición de los elementos a manipular en el área de trabajo no nos obligará a realizar movimientos forzados del tronco con los consiguientes problemas de dolores de espalda. Tanto en el plano vertical como en el horizontal, debemos determinar cuáles son las distancias óptimas que consigan un confort postural adecuado, y que se dan en las figuras 3 y 4 para el plano vertical y el horizontal, respectivamente. (R.C., 2006)

Figura $\mathrm{N}^{\circ}$ 3: Arco de manipulación vertical en el plano sagital

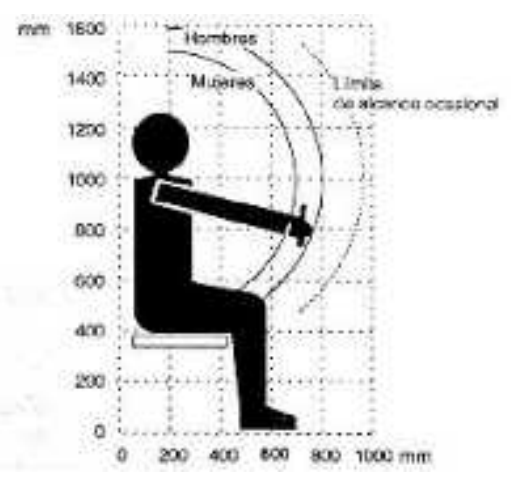

Riesgos ergonómicos en las actividades de docencia y administración 
Figura $\mathrm{N}^{\circ}$ 4: Arco horizontal de alcance del brazo y área de trabajo sobre una mesa (medida en mm)

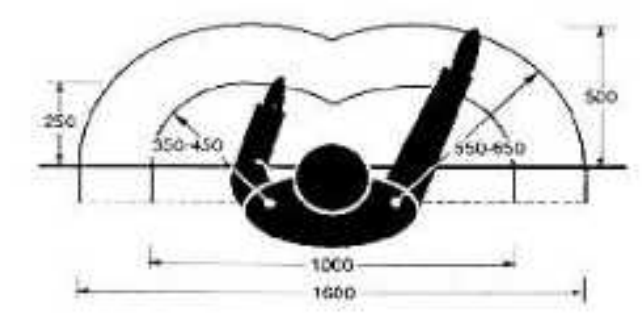

Fuente: Chavarría ,2005.

Elaborado por: Autores de investigación

\section{Silla de trabajo}

Es evidente que la relativa comodidad y la utilidad funcional de sillas y asientos son consecuencia de su diseño en relación con la estructura física y la mecánica del cuerpo humano.

Los usos diferentes de sillas y asientos, y las dimensiones individuales requieren de diseños específicos, no obstante, hay determinadas líneas generales que pueden ayudar a elegir diseños convenientes al trabajo a realizar. La concepción ergonómica de una silla para trabajo de oficina ha de satisfacer una serie de datos y características de diseño. El asiento responderá a las características siguientes:

- Regulable en altura (en posición sentado) margen ajuste entre 380 y 50 mm.

- Anchura entre 400 - $450 \mathrm{~mm}$.

- Profundidad entre 380 y $420 \mathrm{~mm}$.

- Acolchado de $20 \mathrm{~mm}$. recubierto con tela flexible y transpirable.

- Borde anterior inclinado (gran radio de inclinación).

La elección del respaldo se hará en función de los existentes en el mercado, respaldos altos y/o respaldos bajos. (R.C., 2006)

Un respaldo bajo debe ser regulable en altura e inclinación y conseguir el correcto apoyo de las vértebras lumbares. Las dimensiones serán: 
- Anchura 400 - 450 mm.

- Altura 250 - $300 \mathrm{~mm}$.

- Ajuste en altura de 150 - $250 \mathrm{~mm}$.

El respaldo alto debe permitir el apoyo lumbar y ser regulable en inclinación, con las siguientes características:

- Regulación de la inclinación hacia atrás $15^{\circ}$.

- Anchura 300 - 350 mm.

- Altura 450 - $500 \mathrm{~mm}$.

- Material igual al del asiento.

Los respaldos altos permiten un apoyo total de la espalda y por ello la posibilidad de relajar los músculos y reducir la fatiga.

La base de apoyo de la silla debe garantizar una correcta estabilidad de la misma y por ello dispondrá de cinco brazos con ruedas que permitan la libertad de movimiento.

La longitud de los brazos será por lo menos igual a la del asiento (380-450 mm.).

Figura $\mathrm{N}^{\circ}$ 5: Características de diseño de las sillas de trabajo.

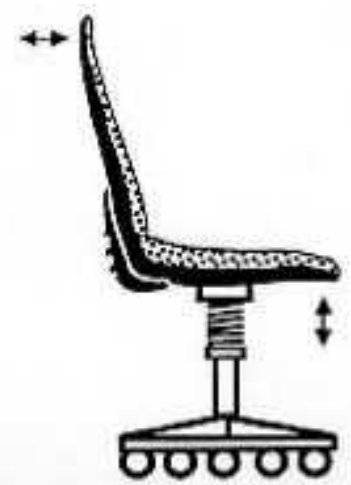

Fuente: Chavarría, 2005.

Elaborado por: Autores de la investigación

\section{Mesa de Trabajo}

Una buena mesa de trabajo debe facilitar el desarrollo adecuado de la tarea; por ello, a la hora de elegir una mesa para trabajos de oficina, deberemos exigir que cumpla los siguientes requisitos: 
- Si la altura es fija, ésta será de aproximadamente $700 \mathrm{~mm}$.

- Si la altura es regulable, la amplitud de regulación estará entre 680 y 700 mm.

- La superficie mínima será de 1, 200 mm de ancho y 800 mm de largo.

- El espesor no debe ser mayor de $30 \mathrm{~mm}$.

- La superficie será de material mate y color claro suave, rechazándose las superficies brillantes y oscuras.

- Permitirá la colocación y los cambios de posición de las piernas.

\section{Reposapiés y apoyabrazos}

"El reposapiés tiene un papel importante, siempre que no se disponga de mesas regulables en altura, ya que permiten, generalmente a las personas de pequeña estatura, evitar posturas inadecuadas". (Guzmán, 2016)

La superficie de apoyo debe asegurar la correcta situación de los pies; las características serán:

- Anchura $400 \mathrm{~mm}$.

- Profundidad $400 \mathrm{~mm}$.

- Altura 50 - $250 \mathrm{~mm}$.

- Inclinación $10^{\circ}$.

Es aconsejable asimismo que la superficie de apoyo de los pies sea de material antideslizante. La utilización de apoyabrazos está indicada en trabajos que exigen gran estabilidad de la mano y en trabajos que no requieren gran libertad de movimiento y no es posible apoyar el antebrazo en el plano de trabajo.

- Anchura 60 - $100 \mathrm{~mm}$.

- Longitud - que permita apoyar el antebrazo y el canto de la mano. 
La forma de los apoyabrazos será plana con los rebordes redondeados.

\section{Niveles mínimos de iluminación para tareas visuales (en Lúmenes)}

Clase Lúmenes

1. Tareas visuales variables y sencillas 250 a 500

2. Tareas visuales continuas y de detalles 500 a 1000

3. Tareas visuales continuas y de precisión 1000 a 2000

4. Trabajos muy delicados y de detalles + de 2000

\section{Conclusiones}

Esta investigación nos ha permitido comprobar la existencia de riesgos ergonómicos dentro del Instituto Tecnológico Superior Paulo Emilio Macías (ITSPEM) en un 95\%.

Los riesgos ergonómicos que con mayor frecuencia se presentan se dan en el puesto de trabajo en lo referente a las posturas, la iluminación inadecuada y del espacio de trabajo.

En cuanto al entorno laboral un factor importante con el que no cuenta la institución es que las sillas no poseen reposapiés.

El 90\% de los docentes y personal administrativo no cuentan con mobiliario ergonómico para el desarrollo de sus actividades.

Se detectaron los factores de riegos ergonómicos que prevalecen en los puestos de trabajos de los docentes y administrativos del ITSPEM, como producto final se realizó una serie de recomendaciones encaminadas a proteger la salud del trabajor.

Con lo que respecta al mobiliario (escritorios y sillas de trabajo) resultó ser uno de los aspectos más deficientes entre los puntos estudiados, ya el 85\%de los trabajadores encuestados respondieron que el mobiliario se encuentra en malas condiciones e incómodos debido a los espacios reducidos con los que se cuentan.

Otro de los puntos observados de mayor relevancia, es que se confirma la importancia del ambiente luminoso y la necesidad de priorizar su evaluación entre los factores físicos del ambiente, que rodea 
los puestos de trabajo que utilizan computadoras, con la finalidad de minimizar los efectos negativos a la visión de los usuarios.

El $85 \%$ del personal estudiado, de acuerdo a los resultados obtenidos demuestran que se desconoce cuál es la postura correcta que se debe adoptar cuando se trabaja frente a una mesa de trabajo más de dos horas continuas durante una jornada laboral.

Como consecuencia del diseño antiergonómico de los 65 puestos de trabajo evaluados, se constata que los problemas de salud más persistentes y que requieren de atención médica periódicamente, son de tipo visual con una incidencia del $62.85 \%$ y músculo esqueléticos (principalmente en zona lumbar) con una incidencia 37.14 entre la población estudiada.

El personal estudiado mostró interés en conocer sobre aspectos ergonómicos, principalmente en posturas.

\section{Bibliografía}

1. ANUIES. (1997. ). Nuevas Tecnologías en la Enseñanza- Aprendizaje. México.: La Academia.

2. Bonilla, R. E. (2001). La Ergonimía en la Gestión de Riesgos de Trabajo. Higiene y Seguridad, 12.

3. Donald, M. (2003). Renueve su oficina. Obtenido de www.prensalibre.com/pl/2003/marzo/18/51717.html171.

4. Guzmán, S. (23 de 10 de 2016). Diseño Orientado al Hombre. Obtenido de http://expgrafica.uma.es/Asignaturas/dis-prod/document/formas.pdf.

5. Hérnandez, G. J. (15 de 08 de 2016). La Importancia de Trabajar con Computadoras en un Ambiente Adecuado. Obtenido de www.reduaeh.mx/campus/icea/publicacion1.

6. Manual de normas técnicas para el diseño ergonómico de puestos con pantallas de visualización. (2005). Obtenido de http://www.mtas.es//insht/practice/pvd.htm.

7. Mondelo P. (2002). Ergonomía 4. El trabajo en oficinas. . México.

8. Montes, H. T. (2000). Elementos de Iluminación en los Centros de Trabajo. Higiene y Seguridad, 15.

9. Ocupacional, I. N. (23 de 10 de 2016). Salud y seguridad. Obtenido de https://www.cdc.gov/spanish/niosh/topics/default.html.

10. R.C., C. (2006). Análisis Ergonómico de los Espacios de Trabajo en Oficinas. España. 
11. Ramirez, C. (2010). Ergonomía y Productividad. México: Limusa.

12. Rodríguez, H. I. (23 de septiembre de 2006). Arqhys. Obtenido de www.arqhys.com/arquitectura/antropometria.html.

13. Rodríguez, H. I. (23 de Septiembre de 2006.). Concepto Ergonomía. Obtenido de www.arqhys.com/: www.arqhys.com/arquitectura/antropometria.html.

14. TeensHealth. (23 de 10 de 2016). Las lesiones provocadas por un esfuerzo repetitivo. Obtenido de http://kidshealth.org/es/teens/rsi-esp.html.

15. Vélez, C. M. (2002). Diseñar desde la ergonomía evita riesgos. España. 